\title{
Inheriting the spirit of a sage: a review of 'The Overarching: A Documenta Celebrating the 120th Anniversary of the Birth of Liang Sicheng'
}

\author{
Yishi Liu*
}

The year 2021 marks the 120th anniversary of the birth of Liang Sicheng. To celebrate this historical event, the School of Architecture of Tsinghua University began preparations in the fall of 2020 by appointing Liu Chang and Wang Nan, two faculty members from the department, to collect and organise various historical materials and exhibits. After 10 months of work, 'The Overarching: A Documenta Celebrating the 120th Anniversary of the Birth of Liang Sicheng' debuted in August 2021 on the 4th floor of Tsinghua University Art Museum (Fig. 1).

According to the Yinzao fashi (《营造法式》), 'Shuowen: ji (极) refers to dong (栋, house), and dong refers to wuji (the apex of the house, 屋极)' (Liang 2013, 44). The name of the exhibit, Dongliang (栋梁, ridge and beam), implies a deep respect for Liang's study of the history of ancient Chinese architecture and its academic contribution. A total of 368 exhibit items (groups), surrounding five themes, were selected. Among them, Liang Sicheng's family background and education served as an important foundation for his future career and thus the first things visitors will see when entering the exhibition hall. The exhibits are arranged according to Liang Sicheng's work and deeds in four fields, i.e., the history of ancient Chinese architecture, urban planning and cultural heritage preservation, architectural creation, and architectural education, forming a total of five exhibition areas, fully reflecting his achievements in various fields

\footnotetext{
*Correspondence: liuyishi@tsinghua.edu.cn

School of Architecture, Tsinghua University, Haidian District, Beijing 10084, China
}

Springer Open

(c) The Author(s) 2021. Open Access This article is licensed under a Creative Commons Attribution 4.0 International License, which permits use, sharing, adaptation, distribution and reproduction in any medium or format, as long as you give appropriate credit to the original author(s) and the source, provide a link to the Creative Commons licence, and indicate if changes were made. The images or other third party material in this article are included in the article's Creative Commons licence, unless indicated otherwise in a credit line to the material. If material is not included in the article's Creative Commons licence and your intended use is not permitted by statutory regulation or exceeds the permitted use, you will need to obtain permission directly from the copyright holder. To view a copy of this licence, visit http://creativecommons.org/licenses/by/4.0/.

of architecture and showing his historical status as the founder and pioneer of the discipline of modern Chinese architecture.

With the assistance of Tsinghua University Art Museum, the exhibit team arranged the five exhibition areas in a $3+2$ format in two connected East and West Exhibition Rooms, with three giant photographs of Liang Sicheng marking the three locations (entrance and east and west ends) directly opposite to the exhibit axis. More than 60 white display cases of different sizes, with a height of less than $700 \mathrm{~mm}$, are arranged under giant photographs of Lang Sicheng; therefore, when standing in front of them, visitors feel 'as if they are attending a lecture by Liang Sicheng in a classroom'. (Fig. 2).

I have visited the exhibit several times and have been tremendously impressed. First, Tsinghua University Art Museum has always exhibited authentic artwork. This exhibition is called 'Documenta Exhibition' because the majority of the exhibits are original items, such as letters (family letters and official letters), certificates (e.g., the honorary doctorate degree Liang received from Princeton University and the State Natural Science Award), and early copies of artwork, not to mention numerous items such as measurements, drawings and renderings. In recent years, I have been engaged in the research of the history of the School of Architecture of Tsinghua University, and for the first time, I saw Liang Sicheng's 


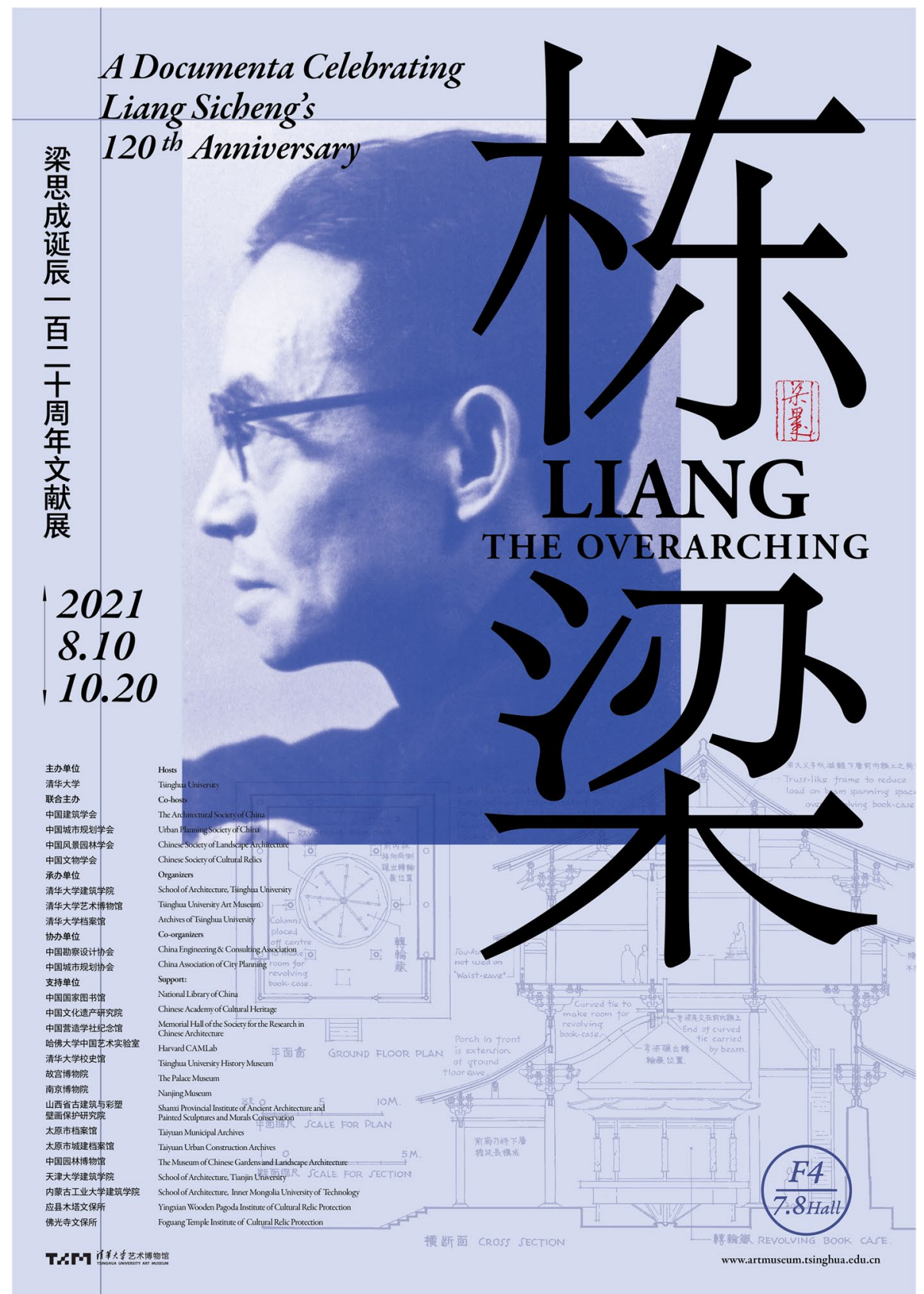

Fig. 1 Exhibition poster (Source: Tsinghua University Art Museum)

handwritten 8-page letter to Mei Yiqi in 1945 proposing the founding of the Department of Architecture. Seeing Liang Sicheng's handwriting and life milestones in detail has made me feel a close connection with him, an experience that transcends time and space. The infectiousness of such visits to view authentic articles of Liang's life is phenomenal.
Second, the exhibits excel in both 'breadth' and 'depth', with a complete coverage. Many items, collected from various public and private institutions in China and abroad, are displayed for the first time. In addition to the more than 200 exhibits (groups) collected by the School of Architecture of Tsinghua University and its affiliated Society for the Study of Chinese Architecture, the exhibition has also received assistance from more than a dozen 


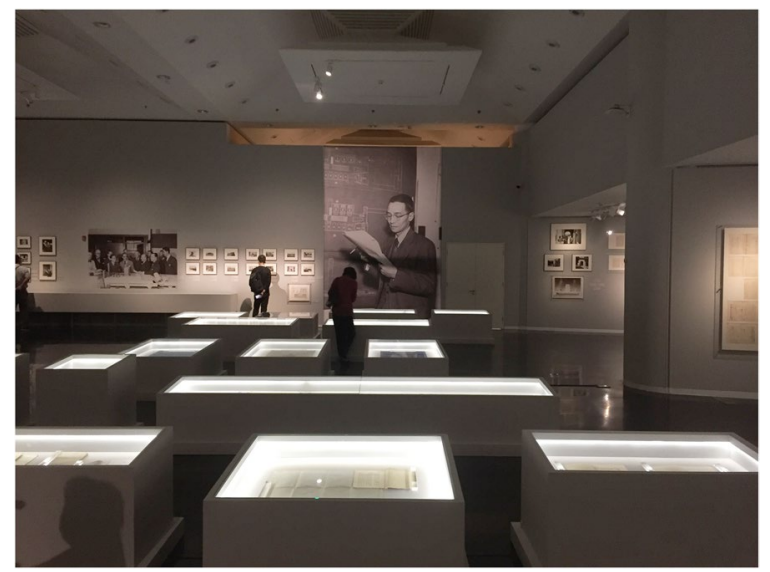

Fig. 2 Photo of the exhibit, including a giant photo of Liang Sicheng showing that he was involved in the design of the United Nations building (1947) and white display cases (Source: the author)

domestic and international institutions, including the National Library of China, the Chinese Academy of Cultural Heritage, Tsinghua University Archives and History Museum, and the Harvard University Chinese Art Media Lab. Liang Sicheng's relatives have also generously provided many private collections for the exhibition. Many of the exhibits are mesmerising and repeatedly viewed by visitors, such as Liang Qichao's letter to Liang Sicheng informing him of the development status of the Department of Architectural Engineering at Northeastern University (1928), a letter from Liang Sicheng and Lin Huiyin to their daughter Liang Zaibing about their experience surveying ancient architectures in Shanxi (1937), and an A0-size watercolour rendering of Dule Temple in Jixian County, and all were provided by organisations or individuals outside Tsinghua University (Figs. 3 and 4).

Third, the exhibits allow the visitors to visualise the spirit of Liang Sicheng at different periods of his life as well as his attitude towards the country, career and life, while feeling his passion. The exhibits include not only letters written by Liang Sicheng to the leaders of the Central Government, giving outspoken advice on the construction of the Monument to the People's Heroes and other construction projects in the capital, but also manuscripts for several popular science articles in the collection Essays of a Humble Craftsman (《拙匠随 笔》) for general readers, with retained hand-written revisions, reflecting his rigour, sincerity and passion for China's architecture. Various scenes in which Liang Sicheng is seen mingling with the faculty and students of the Department of Architecture of Tsinghua University (including a photo with the faculty and students of Tongji University) are the main content of the 'Architectural Education' section. I am particularly touched by a

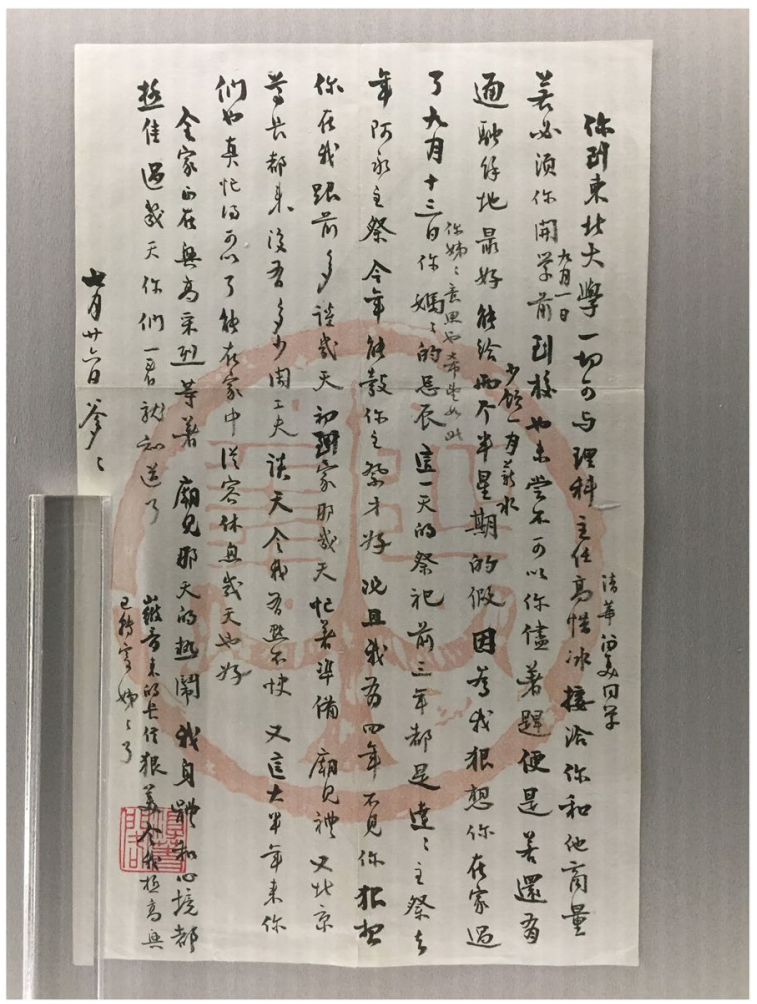

Fig. 3 Letter from Liang Qichao to Liang Sicheng, informing him of the founding of the Department of Architecture and Engineering at Northeastern University and accepting the chairmanship of the department on his behalf (Source: the author)

photograph of Liang Sicheng taken in the Summer Palace in the summer and autumn of 1955 when he just lost his wife, Lin Huiyin, and the 'anti-waste' movement was raging, in which, Liang Sicheng appears calm and composed, reflecting his typical mentality of positiveness and optimism characteristic of his entire life. In fact, this is the time when Liang Sicheng painted for students who matriculated into the Department of Architecture of Tsinghua University in 1955 who were visiting the Summer Palace (Huang 2001, 104-107) (Fig. 5).

Since its opening on August 10, 2021, the exhibition has received tens of thousands of visitors from all walks of life as has generally been well received. Although visitors with different life experiences and intellectual backgrounds have different perceptions of the exhibits, the original intention of 'allowing different visitors to linger and immerse themselves in the exhibition' has been achieved. Nevertheless, the curators are not without regrets. For example, according to Liu Chang, due to the sheer volume of exhibits collected in the past year, it is impossible to present all of them, and the authentic items on display are not yet well protected in optimal conditions (lighting, temperature and humidity). Moreover, 


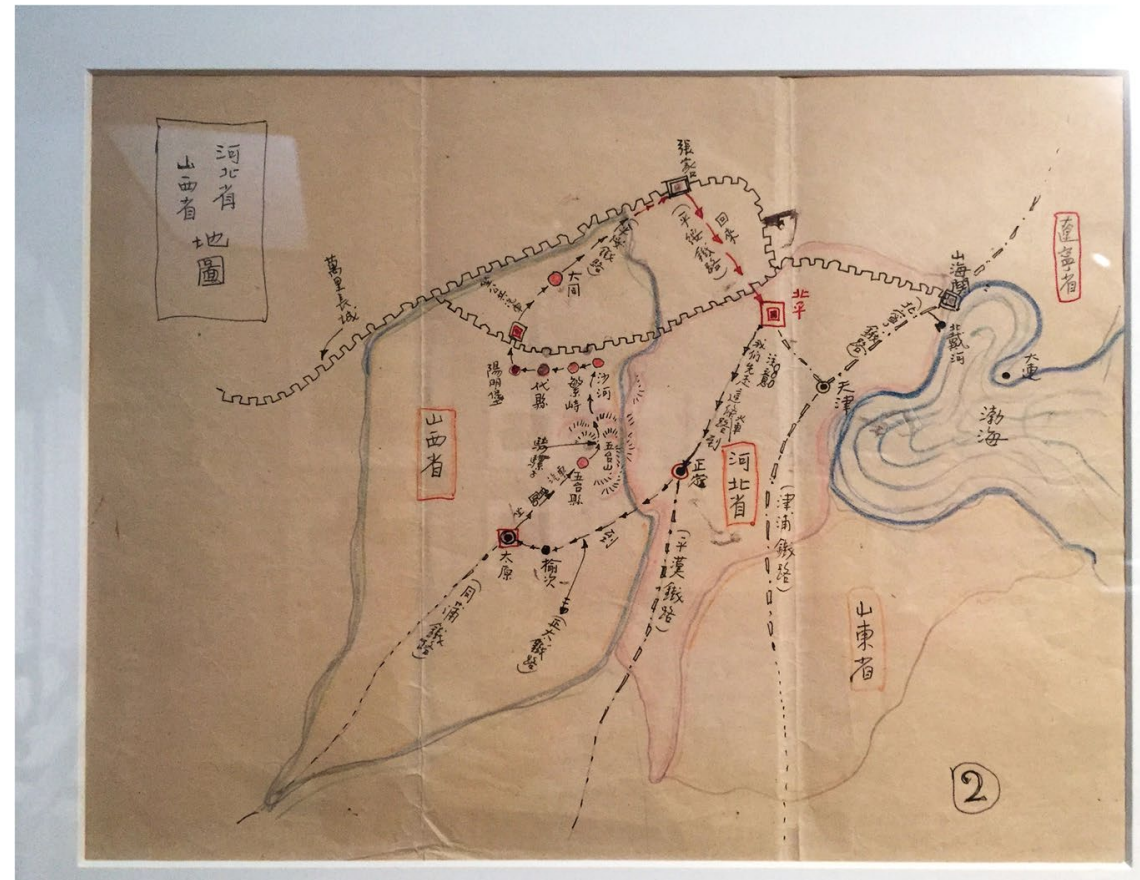

Fig. 4 Letter from Lin Huiyin and Liang Sicheng to Liang Zaibing (age 8years at the time), with a hand-drawn road map related to surveys of ancient architectures in Shanxi and Hebei provinces (Source: the author)

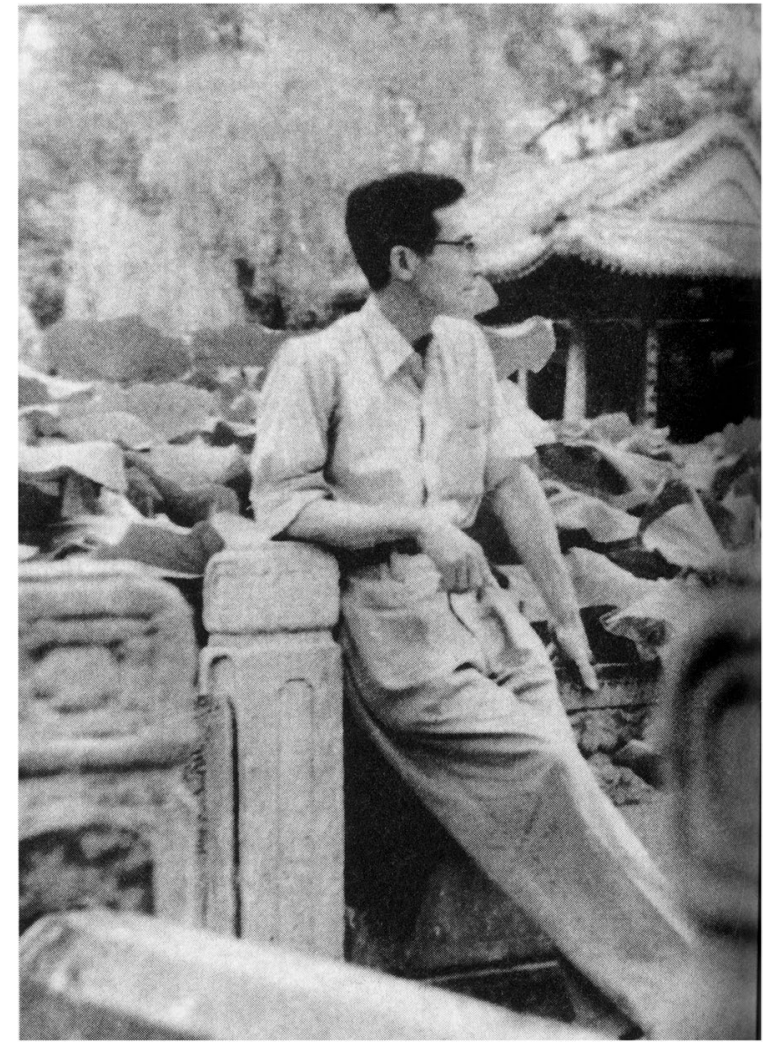

Fig. 5 Photo showing Liang Sicheng in relaxed posture in the Summer Palace in 1955 (Source: Lin 2011, 322) display cases with fixed dimension were ordered; therefore, some newly acquired exhibition items do not fit and are thus unable to be included. I have also heard that it is difficult for elderly visitors to see some content in the display cases because the cases are too low; additionally, the description of some items should be clearer so that the visitors can better appreciate them. For the exhibits regarding the Zhaozhou Bridge (Anji Bridge), except for the stone model (made in the early 1960s, provided by the China Heritage Institute), the rest of the exhibits are pictures of stone quoins and towers taken during field surveys in Sichuan and Kunming, at different times; I learned later that this group of exhibits actually belongs to the category 'non-wooden' structures. There are also some similar small 'exhibition' areas, but they are are prone to be overlooked; as such, the Art Museum provides guided visits, which can also be accessed on line.

The Tsinghua University School of Architecture and Tsinghua University Art Museum will collaborate to include the pictures from all exhibits in a book and publish it in near future, thus significantly expanding the audience. For the 'gems' in the exhibition, three curators have written and published articles that include many priceless pictures (Wang, Liu, and Su 2021).

\section{Acknowledgements}

The author acknowledges the sharing of Professor Liu Chang, the curator of the exhibition on the materials and underpinning logics of the exhibition. 
Author's contributions

The author read and approved the final manuscript.

\section{Funding}

Not applicable.

Availability of data and materials

Not applicable.

\section{Declarations}

Competing interests

The author has no competing interests.

Received: 14 September 2021 Accepted: 24 September 2021

Published online: 01 November 2021
References

Huang, Hui. 2001. "Sinian women de lao huoban." [In memory of our old friend]. In Liang Sicheng xiansheng baisui danchen jinian wenji [A Collection of Articles in memory of the 100th anniversary of the birth of Liang Sicheng], edited by School of Architecture, Tsinghua University, 104-107. Beijing: Tsinghua University Press

Liang, Sicheng. 2013. Yingzao fashi zhushi [Commentary on the treatise of architectural methods]. Beijing: SDX Joint Publishing Company.

Wang, Nan, Chang Liu, and Dan Su. 2021. The recorder of culture and the mirror of history: A review of the Articles in memory of the 100th anniversary of the birth of Liang Sicheng. Architectural Journal 9: 90-115.

Lin, Zhu. 2001. Liang Sicheng, Lin Huiyin and Me [Liang Sicheng Lin Huiyin yu wo]. Beijing: China Youth Publishing House.

\section{Publisher's Note}

Springer Nature remains neutral with regard to jurisdictional claims in published maps and institutional affiliations.

\section{Submit your manuscript to a SpringerOpen ${ }^{\circ}$ journal and benefit from:}

- Convenient online submission

- Rigorous peer review

- Open access: articles freely available online

- High visibility within the field

- Retaining the copyright to your article 